\title{
Covariant electroweak nucleon form factors in a chiral constituent quark model
}

\author{
S. Boffi ${ }^{1,2}$, L. Ya. Glozman ${ }^{1,3}$, W. Klink ${ }^{4}$, W. Plessas ${ }^{3}$, M. Radici ${ }^{1,2}$, R. F. Wagenbrunn ${ }^{1,3}$ \\ ${ }^{1}$ Dipartimento di Fisica Nucleare e Teorica, Università degli Studi di Pavia, I-27100 Pavia, Italy \\ ${ }^{2}$ Istituto Nazionale di Fisica Nucleare, Sezione di Pavia, I-27100 Pavia, Italy \\ ${ }^{3}$ Institut für Theoretische Physik, Universität Graz, A-8010 Graz, Austria \\ ${ }^{4}$ Department of Physics and Astronomy, University of Iowa, Iowa City, IA 52242, USA
}

(February 1, 2008)

\begin{abstract}
Results for the proton and neutron electric and magnetic form factors as well as the nucleon axial and induced pseudoscalar form factors are presented for the chiral constituent quark model based on Goldstone-boson-exchange dynamics. The calculations are performed in a covariant framework using the point-form approach to relativistic quantum mechanics. The direct predictions of the model yield a remarkably consistent picture of the electroweak nucleon structure.
\end{abstract}

12.39-x; 13.10.+q; 14.20.Dh

The nucleon as a composite system of strongly interacting quarks and gluons has ultimately to be described in all aspects on the basis of quantum chromodynamics (QCD). This is a difficult task, especially at low and intermediate energies, where QCD cannot be solved perturbatively. In this regime, one of the primary issues is to identify the proper effective degrees of freedom. In particular, one has to respect the spontaneous breaking of chiral symmetry as an essential low-energy property of QCD. It leads among other things to the concepts of constituent quarks and Goldstone bosons. Recently, a chiral quark model incorporating these degrees of freedom has been especially successful in describing the spectroscopy of baryons with light and strange flavours [1].

Beyond spectroscopy, further stringent tests of any constituent quark model (CQM) consist in the proton and neutron electromagnetic form factors, $G_{E}$ and $G_{M}$, observed in elastic electron-nucleon scattering (for a review of recent data, see Ref. [2]). Further important constraints are furnished by the nucleon weak form factors, i.e. the axial form factor $G_{A}$ and the induced pseudoscalar form factor $G_{P}$. They reflect the structure of the nucleons as probed by an axial vector field in processes such as beta decay, muon capture, and pion production. In contrast to the electromagnetic form factors, the weak form factors involve a combination of the proton and neutron wave functions.

Due to their large kinetic energy, constituent quarks require an essentially relativistic quantum mechanical treatment. For CQMs one can incorporate relativity into a quantum theory with a finite number of degrees of freedom by utilizing relativistic Hamiltonian dynamics (i.e. Poincaré-invariant quantum mechanics) [3]. From the various (unitarily equivalent) forms that are possible when defining the (kinematic) stability subgroup [ $⿴$, we adopt the point form, which has some obvious advantages [5] in our studies. In fact, the four-momentum operators $P^{\mu}$ containing all the dynamics commute with each other and can be diagonalized simultaneously. All other generators of the Poincaré group are not affected by interactions. In particular, the Lorentz generators are interaction-free and make the theory manifestly covariant. Moreover, the electromagnetic current operator $J^{\mu}(x)$ can be written in such a way that it transforms as an irreducible tensor operator under the strongly interacting Poincaré group. Thus the electromagnetic form factors can be calculated as reduced matrix elements of such an irreducible tensor operator in the Breit frame. The same procedure can be applied to the axial current. Once $G_{A}$ is known, $G_{P}$ can be extracted from the longitudinal part of the axial current in the Breit frame.

In this paper, we give a combined presentation of relativistically covariant results for all elastic electroweak form factors of the nucleons as predicted by the specific CQM proposed in Ref. [1]. It relies on a relativistic kinetic energy operator and an instantaneous pairwise linear confinement potential with a strength corresponding to the string tension of QCD. The hyperfine interaction of the constituent quarks is derived from the pseudoscalar Goldstone-boson exchange (GBE) [6]. This kind of dynamics produces a flavor-dependent quark-quark interaction. In the CQM of Ref. [1], only its spin-spin component is utilized, as this has turned out to be the most important part for the hyperfine splitting in the baryon spectra. Indeed, a very reasonable description of the low-energy spectra of all light and strange baryons is achieved with only a few free parameters. In particular, the specific spin-flavor dependence and the sign of the short-range part of the GBE hyperfine interaction produce the correct level orderings of the lowest positive- and negative-parity states, thus remedying a long-standing problem in baryon spectroscopy.

Considering a three-body system in its center-ofmomentum frame with constituent (quark) masses $m_{i}$ and individual 3-momenta $\vec{k}_{i}\left(\sum_{i} \vec{k}_{i}=0\right)$, such an interaction can be introduced through the so-called Bakamjian-Thomas (BT) construction [7], by adding to the free mass operator $M_{0}=\sum_{i} \sqrt{\vec{k}_{i}^{2}+m_{i}^{2}}$ an interaction part $M_{I}$ so that $M=\sqrt{P^{\mu} P_{\mu}}=M_{0}+M_{I}$. Then also the 4-momentum operator gets split into a free part $P_{0}^{\mu}$ and an interaction part $P_{I}^{\mu}: P^{\mu}=P_{0}^{\mu}+P_{I}^{\mu}=M V^{\mu}=$ 
$\left(M_{0}+M_{I}\right) V^{\mu}$. Here $V^{\mu}$ is the 4-velocity of the system, which is not modified by the interaction (i.e., $V^{\mu}=V_{0}^{\mu}$ ). In order to fulfill the Poincaré algebra of 4-momentum operators, the mass operator $M$ with interactions must satisfy the conditions $\left[V^{\mu}, M\right]=0$ and $U(\Lambda) M U^{-1}(\Lambda)=$ $M$, where $U(\Lambda)$ is the unitary operator representing the Lorentz transformation $\Lambda$. In the center-of-momentum frame of the three-body system, the stationary part of the eigenvalue problem $P^{\mu}|\Psi\rangle=p^{\mu}|\Psi\rangle$ can be identified with the eigenvalue problem solved in Ref. [1] for the Hamiltonian $H=\sum_{i} \sqrt{\vec{k}_{i}^{2}+m_{i}^{2}}+H_{I}=H_{0}+H_{I}$. The eigenfunctions of this mass operator describe the threequark system in the center-of-momentum frame. Since the Lorentz boosts and spatial rotations are purely kinematic in the point-form approach, such a wave function can be boosted exactly to the initial and final nucleon states in the Breit frame, where the necessary covariant form factors can be extracted from the corresponding matrix elements without any further approximations.

The current operator is assumed to be a single-particle current operator for point-like constituent quarks. This corresponds to a relativistic impulse approximation but specifically in point form. It is called the point-form spectator approximation (PFSA) because the impulse delivered to the nucleon is different from that delivered to the struck constituent quark. The electromagnetic current matrix elements have the usual form for a point-like Dirac particle, i.e.

$$
\left\langle p_{i}^{\prime}, \lambda_{i}^{\prime}\left|j^{\mu}\right| p_{i}, \lambda_{i}\right\rangle=e_{i} \bar{u}\left(p_{i}^{\prime}, \lambda_{i}^{\prime}\right) \gamma^{\mu} u\left(p_{i}, \lambda_{i}\right),
$$

with $u\left(p_{i}, \lambda_{i}\right)$ the Dirac spinor of quark $i$ with charge $e_{i}$, momentum $p_{i}$, and spin projection $\lambda_{i}$. Such a $j^{\mu}$ is not conserved a-priori but one can always construct a conserved current $\widetilde{j}^{\mu}=j^{\mu}-q^{\mu}\left(q \cdot j / q^{2}\right)$, with $q$ the 4 -momentum transfer. The new added term does not affect the $\mu=0,1,2$ components of the form factors (see Eqs. (位) and (5) below). The axial current matrix elements have the form

$$
\begin{aligned}
& \left\langle p_{i}^{\prime}, \lambda_{i}^{\prime}\left|A_{a}^{\mu}\right| p_{i}, \lambda_{i}\right\rangle= \\
& \quad \bar{u}\left(p_{i}^{\prime}, \lambda_{i}^{\prime}\right)\left[g_{A}^{q} \gamma^{\mu}+\frac{2 f_{\pi}}{\widetilde{Q}^{2}+m_{\pi}^{2}} g_{\pi q} \widetilde{q}^{\mu}\right] \gamma_{5} \frac{1}{2} \tau_{a} u\left(p_{i}, \lambda_{i}\right),
\end{aligned}
$$

where $m_{\pi}$ is the pion mass, $f_{\pi}=93.2 \mathrm{MeV}$ the pion decay constant, and $\widetilde{Q}^{2}=-\widetilde{q}^{2}$, with $\widetilde{q}=p_{i}^{\prime}-p_{i}$ the momentum transferred to a single quark. The quark axial charge is assumed to be $g_{A}^{q}=1$, as for free bare fermions, and $g_{\pi q}$ is identified with the pion-quark coupling constant, with a numerical value as employed in the GBE CQM of Ref. [1]. The use of $\widetilde{q}$ in the pion-pole term of Eq. (2) follows from the momentum given to the constituent quark, in contrast to the momentum $q$ transferred to the whole nucleon.

Following the formalism developed in Ref. [5], the electroweak form factors are obtained in terms of reduced matrix elements $G^{i}$ between nucleon states in the Breit frame. They are given by

$$
\begin{aligned}
& G_{s^{\prime} s}^{i}\left(Q^{2}\right)= \\
& 3 \int d \vec{k}_{1} d \vec{k}_{2} d \vec{k}_{3} d \vec{k}_{1}^{\prime} d \vec{k}_{2}^{\prime} d \vec{k}_{3}^{\prime} \delta\left(\vec{k}_{1}+\vec{k}_{2}+\vec{k}_{3}\right) \delta\left(\vec{k}_{1}^{\prime}+\vec{k}_{2}^{\prime}+\vec{k}_{3}^{\prime}\right) \\
& \times \delta^{3}\left[k_{2}^{\prime}-B^{-1}\left(v_{\text {out }}\right) B\left(v_{\text {in }}\right) k_{2}\right] \delta^{3}\left[k_{3}^{\prime}-B^{-1}\left(v_{\text {out }}\right) B\left(v_{\text {in }}\right) k_{3}\right] \\
& \times \psi_{s^{\prime}}^{*}\left(\vec{k}_{1}^{\prime}, \vec{k}_{2}^{\prime}, \vec{k}_{3}^{\prime} ; \mu_{1}^{\prime}, \mu_{2}^{\prime}, \mu_{3}^{\prime}\right) D_{\lambda_{1}^{\prime} \mu_{1}^{\prime}}^{1 / 2}\left[R_{W}\left(k_{1}^{\prime}, B\left(v_{\text {out }}\right)\right)\right] \\
& \times\left\langle p_{1}^{\prime}, \lambda_{1}^{\prime}\left|J^{\mu}\right| p_{1}, \lambda_{1}\right\rangle \\
& \times D_{\lambda_{1} \mu_{1}}^{1 / 2}\left[R_{W}\left(k_{1}, B\left(v_{\text {in }}\right)\right)\right] \psi_{s}\left(\vec{k}_{1}, \vec{k}_{2}, \vec{k}_{3} ; \mu_{1}, \mu_{2}, \mu_{3}\right) \\
& \times D_{\mu_{2}^{\prime} \mu_{2}}^{1 / 2}\left[R_{W}\left(k_{2}, B^{-1}\left(v_{\text {out }}\right) B\left(v_{\text {in }}\right)\right)\right] \\
& \times D_{\mu_{3}^{\prime} \mu_{3}}^{1 / 2}\left[R_{W}\left(k_{3}, B^{-1}\left(v_{\text {out }}\right) B\left(v_{\text {in }}\right)\right)\right]
\end{aligned}
$$

where $Q^{2}=-q^{2}$, the negative square of the 4-momentum $q$ transferred to the nucleon. In Eq. (3) a summation is understood for repeated indices, and the initial and final 4 -velocities are defined according to the nucleon total momenta in the Breit frame as $M v_{\text {in }}=p_{B}$ and $M v_{\text {out }}=p_{B}^{\prime}$, respectively. $\psi_{s}$ is the center-of-momentum nucleon wave function with $\vec{k}_{i}$ the individual quark momenta, $\mu_{i}$ the spin projections, and $s$ the nucleon total-spin projection. $D^{1 / 2}$ is the standard rotation matrix, $R_{W}$ is the Wigner rotation, and $B(v)$ is a canonical boost of the center-ofmomentum states to the Breit frame with 4 -velocity $v$, where the quark momenta become $p_{i}=B(v) k_{i}$.

Replacing $J^{\mu}$ in Eq. (3) by $j^{\mu}$ of Eq. (11) gives (with $\vec{q}$ along the $\hat{z}$ axis)

$$
\begin{aligned}
& G_{s^{\prime} s}^{0}\left(Q^{2}\right)=G_{E}\left(Q^{2}\right) \delta_{s^{\prime} s}, \\
& G_{s^{\prime} s}^{1}\left(Q^{2}\right)= \pm \frac{Q}{2 M} G_{M}\left(Q^{2}\right) \delta_{s^{\prime}, s \pm 1},
\end{aligned}
$$

while replacing by $A_{a}^{\mu}$ of Eq. (2) gives

$$
\begin{aligned}
& G_{s^{\prime} s}^{1}\left(Q^{2}\right)=\frac{E_{B}}{M} G_{A}\left(Q^{2}\right) \delta_{s^{\prime}, s \pm 1}, \\
& G_{s^{\prime} s}^{3}\left(Q^{2}\right)=\left(G_{A}\left(Q^{2}\right)-\frac{Q^{2}}{4 M^{2}} G_{P}\left(Q^{2}\right)\right) \delta_{s^{\prime} s},
\end{aligned}
$$

with $E_{B}$ the total nucleon energy in the Breit frame.

The results for the proton electromagnetic form factors are shown in Fig. 1, and the corresponding charge radius and magnetic moment are given in Table 1 . The predictions of the GBE CQM obtained in PFSA fall remarkably close to the experimental data; even the trend of the most recent data on the ratio $G_{E}^{p} / G_{M}^{p}$ from TJNAF [8] (filled triangles in the bottom panel of Fig. 1) is reproduced. We emphasize that no additional parameters whatsoever have been introduced to obtain these results, only the quark model wave functions have been used as input into the calculations. In the upper panels of Fig. 1 and in the second column of Table 1 results are shown also for the nonrelativistic impulse approximation (NRIA), i.e. with the standard nonrelativistic form of the current operator and no Lorentz boosts applied to the nucleon wave functions. They are strikingly different from the covariant PFSA results. Consequently 
the effects of relativity appear most essential for the description of the nucleon form factors, even at vanishing momentum transfers.

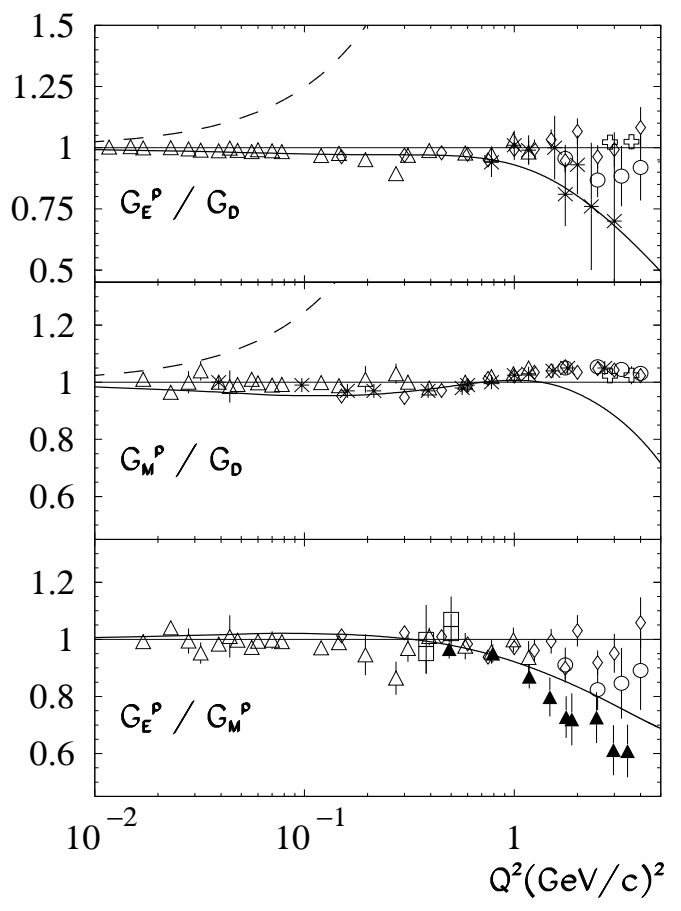

FIG. 1. Proton electric and magnetic form factors. Top and middle: Ratios of $G_{E}^{p}$ and $G_{M}^{p}$ to the standard dipole parametrization $G_{D}$. Bottom: Ratio of $G_{E}^{p}$ to $G_{M}^{p}$. PFSA predictions of the GBE CQM (solid lines) are compared to NRIA results (dashed lines) and to experiment. In the top and middle panels the experimental data are from Ref. 99. In the bottom panel recent data from TJNAF [8] (filled triangles) are shown together with various older data points (see Ref. [8] and refs. therein). All the ratios are normalized to 1 at $Q^{2}=0$.

The results for the neutron electromagnetic structure are shown in Fig. 2 and again in Table 1 . The quality of the predictions of the GBE CQM is about the same as in the proton case, the effects of relativity are similarly important. The neutron electric form factor and its charge radius can be described reasonably well only with a realistic three-quark wave function. For example, in the top panel of Fig. 2 and in Table 1 we give also the results for the case with the confinement potential only, i.e. using $\mathrm{SU}(6)$ symmetric wave functions. It is immediately evident that $G_{E}^{n}$ is essentially driven by the combined effects of small mixed-symmetry components in the neutron wave function (which are induced only by the hyperfine interaction) and Lorentz boosts; the same is true for the neutron charge radius (see Table 1 ).

The nucleon axial form factor $G_{A}$ and the induced pseudoscalar form factor $G_{P}$ are shown in Fig. 3, and the axial radius $\left\langle r_{A}^{2}>^{\frac{1}{2}}\right.$ as well as the axial charge $g_{A}$ are given in Table 1 . In the top panel of Fig. 3 the
TABLE I. Proton and neutron charge radii as well as magnetic moments and nucleon axial radius as well as axial charge. Predictions of the GBE CQM in PFSA (third column), in NRIA (fourth column), and with the confinement interaction only (last column).

\begin{tabular}{ccccc}
\hline \hline & Exp. & PFSA & NRIA & Conf. \\
\hline$r_{p}^{2}\left[\mathrm{fm}^{2}\right]$ & $0.780(25) \sqrt[16]{17}$ & 0.81 & 0.10 & 0.37 \\
$r_{n}^{2}\left[\mathrm{fm}^{2}\right]$ & $-0.113(7) \sqrt[17]{12}$ & -0.13 & -0.01 & -0.01 \\
$\mu_{p}$ [n.m.] & $2.792847337(29) \sqrt{12}$ & 2.7 & 2.74 & 1.84 \\
$\mu_{n}$ [n.m.] & $-1.91304270(5)[12$ & -1.7 & -1.82 & -1.20 \\
$<r_{A}^{2}>^{\frac{1}{2}}[\mathrm{fm}]$ & $0.635(23) \sqrt[13]{13}$ & 0.53 & 0.36 & 0.43 \\
$g_{A}$ & $1.255 \pm 0.006[12]$ & 1.15 & 1.65 & 1.29 \\
\hline \hline
\end{tabular}

$G_{A}$ predictions of the GBE CQM in PFSA are compared to experimental data, which are presented assuming the common dipole parameterization with the axial charge $g_{A}=1.255 \pm 0.006$, as obtained from $\beta$-decay experiments [12], and three different values for the nucleon axial mass $M_{A}$. Again a remarkable coincidence of theory and experiment is detected; only at $Q^{2}=0$ does the PFSA calculation underestimate the experimental value of $g_{A}$ and, consequently, also the axial radius. In contrast, both the NRIA results and also the results from a calculation with the relativistic axial current of Eq. (2) but no boosts on the wave functions fall tremendously short. Again the inclusion of all relativistic effects, in order to produce a covariant result, appears most essential.

The PFSA predictions of the GBE CQM for the induced pseudoscalar form factor $G_{P}$ also fall readily on the available experimental data. For this result the pionpole term occurring in the axial current of Eq. (2) turns out to be most important, especially at low $Q^{2}$. This is clearly seen by a comparison of the solid curve in the lower panel of Fig. 3 with the results obtained without the pion-pole term. It follows that at least for low $Q^{2}$ values the role of pions is essential. It is also remarkable that the coincidence of the PFSA predictions with experiment is obtained by using the same value of the quark-pion coupling constant, $g_{\pi q}^{2} / 4 \pi=0.67$, in Eq. (2) as employed in the GBE CQM of Ref. [1].

In summary, the chiral constituent quark model based on GBE dynamics predicts all observables of the electroweak nucleon structure in a consistent manner. The covariant results calculated in the framework of pointform relativistic quantum mechanics always fall rather close to the available experimental data. This indicates that a quark model using the proper low-energy degrees of freedom may be capable of providing a reasonable description also of other (dynamical) phenomena, in addition to a satisfactory description of spectroscopy. Nevertheless, with regard to the electroweak form factors discussed here, a detailed comparison with the experimental data suggests that there is still room for quantitative improvement, e.g. by considering two-body currents or effects from a possible constituent quark structure. 

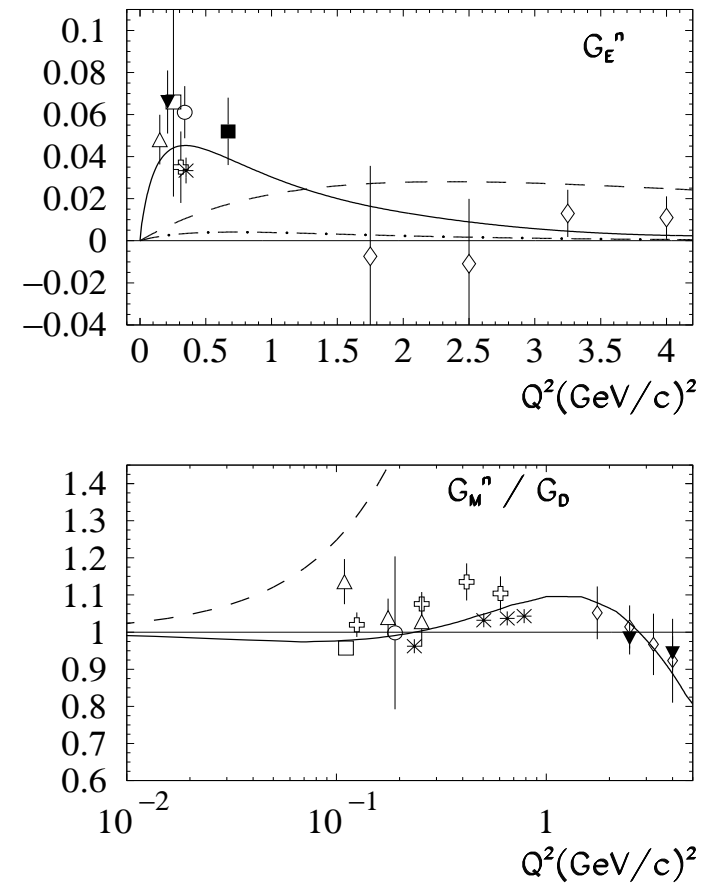

FIG. 2. Neutron electric and magnetic form factors. Top: $G_{E}^{n}$. Bottom: Ratio of $G_{M}^{n}$ to the standard dipole parametrization $G_{D}$, normalized to 1 at $Q^{2}=0$. Solid and dashed lines as in Fig. 1; the dot-dashed line represents the PFSA results for the case with confinement only. Experimental data are from Ref. [10] (top) and Ref. [11] (bottom).

[1] L.Ya. Glozman, W. Plessas, K. Varga and R.F. Wagenbrunn, Phys. Rev. D 58, 094030 (1998).

[2] G.G. Petratos, Nucl. Phys. A 666\&667, 61c (2000).

[3] P.A.M. Dirac, Rev. Mod. Phys. 21, 392 (1949).

[4] S.N. Sokolov and A.N. Shathny, Theor. Mat. Phys. 37, 1029 (1978); H. Leutwyler and J. Stern, Ann. Phys. (N.Y.) 112, 94 (1978); B.D. Keister and W.N. Polyzou, Adv. Nucl. Phys. 20, 225 (1991).

[5] W.H. Klink, Phys. Rev. C 58, 3587 (1998).

[6] L.Ya. Glozman and D.O. Riska, Phys. Rep. 268, 263 (1996).

[7] B. Bakamjian and L.H. Thomas, Phys. Rev. 92, 1300 (1953).

[8] M.K. Jones et al., Phys. Rev. Lett. 84, 1398 (2000).

[9] L. Andivahis et al., Phys. Rev. D 50, 5491 (1994); R.C. Walker et al., Phys. Rev. D 49, 5671 (1994); A.F. Sill et al., Phys. Rev. D 48, 29 (1993); G. Höhler et al., Nucl. Phys. B 114, 505 (1976); W. Bartel et al., Nucl. Phys. B 58, 429 (1973).

[10] T. Eden et al., Phys. Rev. C 50, R1749 (1994); M. Meyerhoff et al., Phys. Lett. B 327, 201 (1994); A. Lung et al., Phys. Rev. Lett. 70, 718 (1993); C. Herberg et al.,
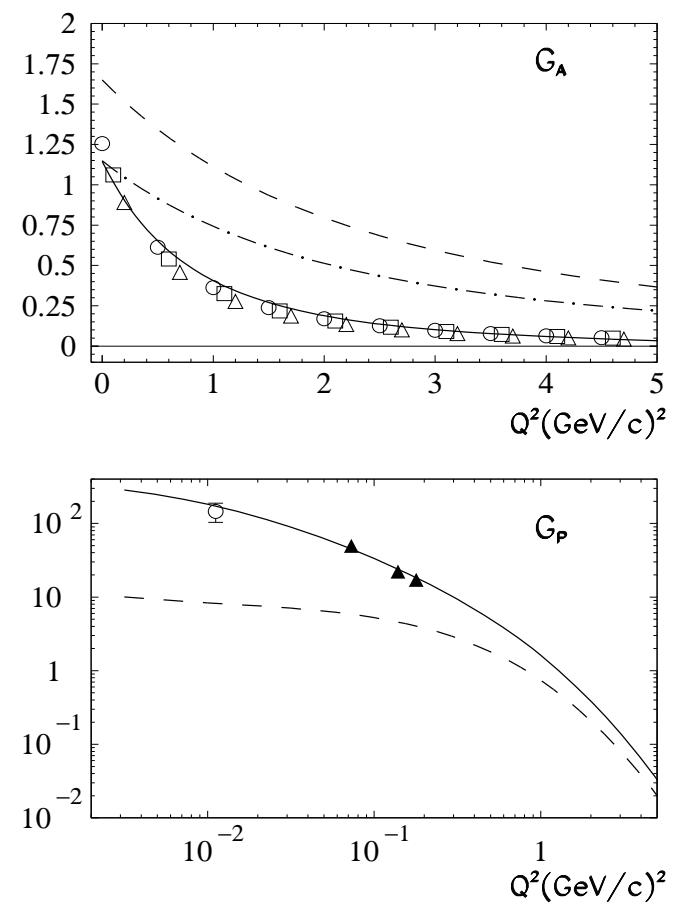

FIG. 3. Nucleon axial and induced pseudoscalar form factors $G_{A}$ and $G_{P}$, respectively. The PFSA predictions of the GBE CQM are always represented by solid lines. In the top panel a comparison is given to the NRIA results (dashed) and to the case with a relativistic current operator but no boosts included (dot-dashed); experimental data are shown assuming a dipole parameterization with the axial mass value $M_{A}$ deduced from pion electroproduction (world average: squares, Mainz experiment 13]: circles) and from neutrino scattering 14. (triangles). In the bottom panel the dashed line refers to the calculation of $G_{P}$ without any pion-pole contribution. The experimental data are from Ref. [15].

Eur. Phys. J. A 5, 131 (1999); I. Passchier et al., Phys. Rev. Lett. 82, 4988 (1999); D. Rohe et al., Phys. Rev. Lett. 83, 4257 (1999); M. Ostrick et al., Phys. Rev. Lett. 83, 276 (1999); J. Becker et al., Eur. Phys. J. A 6, 329 (1999).

[11] P. Markowitz et al., Phys. Rev. C48, R5 (1993); S. Rock et al., Phys. Rev. Lett. 49, 1139 (1982); E.E.W. Bruins et al., Phys. Rev. Lett. 75, 21 (1995); H. Gao et al., Phys. Rev. C50, R546 (1994); A. Lung et al., Phys. Rev. Lett. 70, 718 (1993); H. Anklin et al., Phys. Lett. B336, 313 (1994); H. Anklin et al., Phys. Lett. B428, 248 (1998).

[12] D.E. Groom et al., Eur. Phys. J. C15, 1 (2000).

[13] A. Liesenfeld et al., Phys. Lett. B 468, 20 (1999).

[14] T. Kitagaki et al., Phys. Rev. D28, 436 (1983).

[15] G. Bardin et al., Phys. Lett. B 104, 320 (1981); Seonho Choi et al., Phys. Rev. Lett. 71, 3927 (1993).

[16] K. Melnikov and T. van Ritbergen, Phys. Rev. Lett. 84, 1673 (2000).

[17] S. Kopecky, P. Riehs, J.A. Harvey and N.W. Hill, Phys. Rev. Lett. 74, 2427 (1995). 